\title{
PAPER \\ Adaptive Iterative Decoding of Finite-Length Differentially Encoded LDPC Coded Systems with Multiple-Symbol Differential Detection
}

\author{
Yang YU ${ }^{\dagger a)}$, Student Member, Shiro HANDA ${ }^{\dagger}$, Fumihito SASAMORI ${ }^{\dagger}$, and Osamu TAKYU ${ }^{\dagger}$, Members
}

\begin{abstract}
SUMMARY In this paper, through extrinsic information transfer (EXIT) band chart analysis, an adaptive iterative decoding approach (AIDA) is proposed to reduce the iterative decoding complexity and delay for finite-length differentially encoded Low-density parity-check (DE-LDPC) coded systems with multiple-symbol differential detection (MSDD). The proposed AIDA can adaptively adjust the observation window size (OWS) of the MSDD soft-input soft-output demodulator (SISOD) and the outer iteration number of the iterative decoder (consisting of the MSDD SISOD and the LDPC decoder) instead of setting fixed values for the two parameters of the considered systems. The performance of AIDA depends on its stopping criterion (SC) which is used to terminate the iterative decoding before reaching the maximum outer iteration number. Many SCs have been proposed; however, these approaches focus on turbo coded systems, and it has been proven that they do not well suit for LDPC coded systems. To solve this problem, a new SC called differential mutual information (DMI) criterion, which can track the convergence status of the iterative decoding, is proposed; it is based on tracking the difference of the output mutual information of the LDPC decoder between two consecutive outer iterations of the considered systems. AIDA using the DMI criterion can adaptively adjust the out iteration number and OWS according to the convergence situation of the iterative decoding. Simulation results show that compared with using the existing SCs, AIDA using the DMI criterion can further reduce the decoding complexity and delay, and its performance is not affected by a change in the LDPC code and transmission channel parameters.

key words: adaptive iterative decoding, low-density parity-check (LDPC) codes, differential encoded LDPC coded systems, multiple-symbol differential detection, extrinsic information transfer (EXIT) band chart
\end{abstract}

\section{Introduction}

Low-density parity-check (LDPC) codes have been proved to be a class of capacity-approaching codes over additive white Gaussian noise (AWGN) channels with coherent detection. However, since the performance of coherent detection relies on an accurate phase tracking and the reliable estimation of channel state information, coherent detection becomes expensive or infeasible in some cases. Therefore, differential encoded LDPC (DE-LDPC) coded systems with differential detection, which circumvent the need for phase tracking and channel estimation, have attracted a lot of attention [1]-[4].

It is well known that conventional differential detection has considerably worse performance than coherent de-

\footnotetext{
Manuscript received July 19, 2012.

Manuscript revised October 29, 2012.

${ }^{\dagger}$ The authors are with the Faculty of Engineering, Shinshu University, Nagano-shi, 380-8553 Japan.

a)E-mail: 10st221g@ shinshu-u.ac.jp

DOI: 10.1587/transcom.E96.B.847
}

tection. Multiple-symbol differential detection (MSDD) [5] has been proven to be an effective approach to compensate for this performance loss. For serially concatenated coded systems consisting of a channel encoder and a differential encoder, an iterative decoding scheme based on MSDD was proposed in [6]. In this scheme, the soft-input soft-output demodulator (SISOD) with MSDD (called MSDD SISOD in the following) is used as the inner decoder, and the iterative decoding is performed between the MSDD SISOD and the outer decoder. The process of this demodulator-decoder iteration is denoted by the outer iteration in this paper. It was shown that this iterative MSDD scheme can achieve a large performance improvement compared with conventional differential detection. Up to now, the iterative MSDD scheme has been widely studied, such as iterative MSDD for turbo coded systems [7], for cooperative communication systems [8] and for spatial division multiple access systems [9]. For DE-LDPC coded systems, we investigated the performance of the iterative MSDD scheme in [10].

In general, to achieve excellent performance, the iterative MSDD scheme requires relatively large values of the observation window size (OWS) of MSDD and the outer iteration number between the MSDD SISOD and the outer decoder. However, MSDD with a large OWS will produce a high complexity of MSDD SISOD and cause a long decoding delay when the outer iteration number is also set to a large value, since the complexity of MSDD is exponentially increased with the OWS. On the other hand, at low signal to noise ratios (SNRs), very little performance improvement is achieved by increasing the outer iteration number and using a large OWS. Moreover, at high SNRs, most of the successful decodings can be achieved in the first few iterations with the small OWS. This means that a large outer iteration number and a large OWS are unnecessary in the two SNR regions. In general, due to the fact that the SNR is unknown at the receiver, it is necessary to propose an adaptive iterative decoding approach (AIDA) to adjust the OWS and the outer iteration number adaptively in a changing SNR environment for the coded systems using iterative MSDD.

To adaptively adjust the OWS, an approach based on an analysis of the iterative decoding process using the EXIT chart technique [11] was proposed for "Turbo DPSK" systems [7]. In this approach, the OWS is gradually increased by using a look-up table, which is designed based on a designed iterative decoding trajectory obtained from 
the EXIT chart. Using this approach, the complexity of iterative MSDD can be reduced. However, the design of the OWS table is based on assuming that the iterative decoding trajectory is well matched with the EXIT functions of the two component decoders of the iterative decoder, which is valid for using codes with infinite-length but not for using codes with finite-length [12]. Therefore, the approach of [7] is not suitable for using finite-length LDPC codes. Whereas in practical systems, LDPC codes are generally restricted to blocks of a few hundred to a few thousand code bits.

On the other hand, to adjust the outer iteration number adaptively, a stopping criterion (SC), which is used to judge whether the iterative decoding should be terminated or not, is needed in AIDA. Up to now, many SCs have been proposed to terminate the iteration early to prevent unnecessary iterations of the iterative decoding, such as the cross entropy (CE) criterion [13], the sign-change-ratio (SCR) criterion [14], the sign difference ratio (SDR) criterion [15] and the mean-estimate (ME) criterion [16]. All these criteria were proposed for turbo coded systems and have been proven to be able to reduce the iteration number significantly with little performance loss by comparing a predefined threshold at high SNRs, where successful decodings are usually achieved in the first a few iterations. In [17], the above criteria were modified to stop the iteration at low and high SNRs for turbo coded systems by designing another stopping threshold for low SNRs based on the decoding threshold obtained by the EXIT chart.

Although so many SCs have been proposed for turbo coded systems, little attention has been paid to the iterative decoding of serially concatenated LDPC coded systems. In contrast to turbo codes, LDPC codes can detect successful decoding to stop the iteration by checking the parity check constraints of LDPC codes. Therefore, serially concatenated LDPC coded systems are more concerned with the performance of the SC for uncorrectable decodings. In order to satisfy this requirement, stopping rules and thresholds of these existing SCs should be redefined. However, the analysis results of Sects. 3.3 and 4 show that the redefined stopping rules and thresholds are only suitable for a certain SNR region, which causes significant performance loss at other SNRs. It is also shown that they need to be redefined when the LDPC code and transmission channel parameters change.

In this paper, we consider finite-length DE-LDPC coded systems with MSDD. By analyzing the convergence behavior of the considered systems by the EXIT band chart, which is a generalized EXIT chart for analyzing the finitelength coded systems [12], an AIDA scheme which can adaptively adjust the OWS of the MSDD SISOD and the outer iteration number of the iterative decoder is proposed. In AIDA, the OWS and the outer iteration number are adaptively adjusted by using a SC to judge whether the iterative decoding converges or not. To circumvent the disadvantages of the existing SCs, a new SC which we call differential mutual information (DMI) criterion is proposed for tracking the convergence status of the iterative decoding by track- ing the difference of the output mutual information of the LDPC decoder between two consecutive outer iterations of the considered systems. Simulation results show that AIDA with the proposed DMI criterion can significantly reduce the iterative decoding complexity and delay of the considered systems at all SNRs. Moreover, compared with the existing SCs, it is proved that the DMI criterion is more effective for the considered systems in terms of reducing the average number of outer iterations, performance loss and robustness.

We note that the most closely related work to the DMI criterion presented in this paper is [18], which has proposed an early stopping approach based on the mutual information improvement for turbo-decoding-aided hybrid automatic repeat request (HARQ) schemes. The similarities and differences between [18] and our work will be highlighted in detail in Sects. 3.4 and 4.2.

The rest of this paper is organized as follows. In Sect. 2, the system model and its EXIT band chart analysis are introduced. In Sect. 3, we propose the AIDA scheme, and propose the new SC named DMI after discussing the existing $\mathrm{SCs}$ for the considered systems. Then, the system performance is analyzed using computer simulations in Sect. 4. Finally, Sect. 5 concludes the paper.

\section{System Model and Its EXIT Band Chart Analysis}

\subsection{System Model}

The system model is shown in Fig. 1. A random message bit sequence $\mathbf{b}=\left\{b_{1}, b_{2}, \ldots, b_{K}\right\}, b_{i} \in\{0,1\}$ is first encoded by a rate $K / N$ LDPC encoder to a code sequence $\mathbf{c}=\left\{c_{1}, c_{2}, \ldots, c_{N}\right\}, c_{i} \in\{0,1\}$. The code sequence is then mapped to an $M$-ary PSK symbol sequence $\mathbf{x}=$ $\left\{x_{1}, x_{2}, \ldots, x_{N / m}\right\}, x_{i} \in\left\{e^{j 2 \pi i / M} \mid i=0,1, \ldots, M\right\}$, where $m$ is the number of bits of each $M$-ary PSK symbol. The sequence $\mathbf{x}$ is then differentially encoded to a sequence $\mathbf{s}=$ $\left\{s_{0}, s_{1}, \ldots, s_{N / m}\right\}$, where $s_{k}$ is given by $s_{k}=x_{k} s_{k-1} . s_{0}$ is a reference symbol and is known by the demodulator. In this paper, $s_{0}$ is set to 1 . Different from general serially concatenated codes, the considered systems omit the interleaver between the encoder and the modulator to reduce the complexity of the system, because LDPC codes have an inherent interleaving nature since their parity check matrixes are randomly constructed with a high degree of sparsity [19].

The differential encoded sequence $\mathbf{s}$ is transmitted to

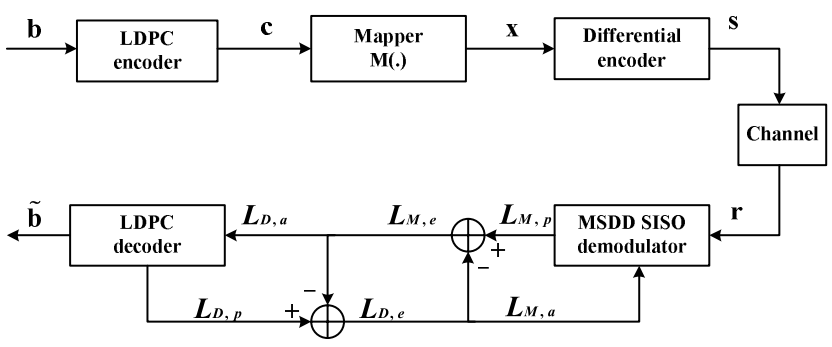

Fig. 1 System model. 
the receiver through AWGN channels. With differential detection, the received discrete-time baseband signal, at time $k$, can be written as

$$
r_{k}=s_{k} e^{j \theta_{k}}+n_{k},
$$

where $\theta_{k}$, which is uniformly distributed over $[0,2 \pi)$, is the unknown phase introduced by the channel, and $n_{k}$ is a sample of a zero mean complex Gaussian noise with variance $\sigma_{n}^{2}$.

At the receiver, the received signals are iteratively decoded by mutually exchanging soft information between the MSDD SISOD and the LDPC decoder. At each outer iteration, the MSDD SISOD produces the a posteriori information $L_{M, p}$ based on the received signals and the a priori information $L_{M, a}$ provided by the LDPC decoder, and produces the extrinsic information $L_{M, e}$ by subtracting $L_{M, a}$ from $L_{M, p}$. Then $L_{M, e}$ is passed to the LDPC decoder as the a priori information $L_{D, a}$. Based on $L_{D, a}$, the LDPC decoder performs a number of inner iterations, and makes a tentative hard decision. If the hard decision is determined to be a legitimate codeword by checking the parity check constraints, the iterative decoding will be terminated. Otherwise, the extrinsic information $L_{D, e}$ of the LDPC decoder, which is obtained in a similar way to $L_{M, e}$, will be fed back to the MSDD SISOD as the a priori information $L_{M, a}$ for the next outer iteration. This process is repeated until the predefined maximum outer iteration number is reached or a legitimate codeword is found.

\subsection{MSDD SISOD}

Here we assume that the OWS of the MSDD SISOD is $S$, and $\theta_{k}$ remains constant over the entire received sequence, i.e., $\theta_{k}=\theta$. The received sequence is divided into subblocks of $S$ symbols each in such a way that the subblocks overlap in one symbol. That is, the number of subblocks is $(N / m) /(S-1)$. For the $k$ th subblock, we can rewrite (1) in the following vector form

$$
\mathbf{r}_{k}=\mathbf{s}_{k} e^{j \theta}+\mathbf{n}_{k},
$$

where $\mathbf{r}_{k}=\left[r_{k, 0}, r_{k, 1}, \ldots, r_{k, S-1}\right]^{T}, \mathbf{s}_{k}=\left[s_{k, 0}, s_{k, 1}, \ldots, s_{k, S-1}\right]^{T}$, $\mathbf{n}_{k}=\left[n_{k, 0}, n_{k, 1}, \ldots, n_{k, S-1}\right]^{T}$, and the superscript ' $T$ ' denotes the transpose operation.

Let $\mathbf{c}_{k}=\left[c_{k, 1}, c_{k, 2}, \ldots, c_{k, m(S-1)}\right]^{T}$ denote the code bits corresponding to the $k$ th subblock of the received symbols. For the sake of clarity, we drop the index $k$ in the following. Based on the maximum a posteriori principle, the MSDD SISOD generates the a posteriori probability (APP) of the code bit $c_{i}$, written in terms of $\log$-likelihood ratio (LLR)

$$
L_{M, p}=\log \frac{\sum_{\mathbf{s}: c_{i}=0} p(\mathbf{r} \mid \mathbf{s}) \prod_{j=1}^{m(S-1)} p\left(c_{j}\right)}{\sum_{\mathbf{s}: c_{i}=1} p(\mathbf{r} \mid \mathbf{s}) \prod_{j=1}^{m(S-1)} p\left(c_{j}\right)},
$$

where the sums in the numerator and denominator are taken over all sequences $\mathbf{s}$ corresponding to the sequences $\mathbf{c}$ whose bit in position $i$ is the value 0 or 1 , respectively. $p\left(c_{j}\right)$ is the a priori probability provided by the LDPC decoder. $p(\mathbf{r} \mid \mathbf{s})$ is the conditional probability density function (PDF) of $\mathbf{r}$ given $\mathbf{s}$. For AWGN channels, $p(\mathbf{r} \mid \mathbf{s})$ is given by [5]

$$
\begin{aligned}
p(\mathbf{r} \mid \mathbf{s})=\frac{1}{\left(2 \pi \sigma_{n}^{2}\right)^{S}} \exp \left[-\frac{1}{2 \sigma_{n}^{2}}\right. & \left.\sum_{i=0}^{S-1}\left(\left|r_{i}\right|^{2}+\left|s_{i}\right|^{2}\right)\right] \\
& \times I_{0}\left(\frac{1}{\sigma_{n}^{2}}\left|\sum_{i=0}^{S-1} r_{i} s_{i}^{*}\right|\right),
\end{aligned}
$$

where $I_{0}(\cdot)$ is the zero-order modified Bessel function of the first kind, and the superscript ' $*$ ' denotes complex conjugation.

\subsection{EXIT Band Chart Analysis of Finite-Length DE- LDPC Coded Systems with MSDD}

The convergence behavior of the iterative decoding of serially concatenated systems can be visualized and predicted by EXIT chart analysis [11]. However, it is known that the analysis results of the EXIT chart are accurate for infinitelength codes, but not for finite-length codes. Whereas in practical systems, LDPC codes are generally limited to short or medium length with a few hundred or thousand bits. In [12], an EXIT band chart, which is a convergence analysis approach using an EXIT curve band instead of a single EXIT curve as in EXIT chart, was proposed for finite-length turbo decoding. In this section, we extend this approach to finite-length DE-LDPC coded systems with MSDD.

In EXIT band chart, the transfer characteristics of component decoders are characterized by their EXIT functions for each random channel realization. Let [s] denote the seed of the channel realization, and let $I_{L_{M, e}^{[s]}}$ and $I_{L_{M, a}^{[s]}}\left(I_{L_{D, e}^{[s]}}\right.$ and $\left.I_{L_{D, a}^{[s]}}\right)$ be mutual informations between the transmitted coded bits and the LLRs at the output and the input of MSDD SISOD (LDPC decoder, respectively). For a given [s], the EXIT function of the MSDD SISOD over AWGN channels is defined as

$$
I_{L_{M, e}^{[s]}}=T_{1}^{[s]}\left(I_{L_{M, a}^{[s]}}, \frac{E_{b}}{N_{0}}\right),
$$

where $\frac{E_{b}}{N_{0}}$ is the SNR of the channel. Similarly, for a given [s], the EXIT function of the LDPC decoder is defined as

$$
I_{L_{D, e}^{[s]}}=T_{2}^{[s]}\left(I_{L_{D, a}^{[s]}}\right) .
$$

The mutual information between the transmitted coded bits $C$ and the corresponding LLR values $L$ is calculated as [11]

$$
\begin{aligned}
I_{L}=I(L ; C)= & \frac{1}{2} \sum_{c=0,1} \int_{-\infty}^{\infty} p_{L}(l \mid c) \\
& \cdot \log _{2} \frac{2 p_{L}(l \mid c)}{p_{L}(l \mid c=0)+p_{L}(l \mid c=1)} d l,
\end{aligned}
$$

where $p_{L}(l \mid c)$ is the conditional PDF of the LLR values $L$ given $c \in\{0,1\}$, and $0 \leqslant I_{L} \leqslant 1$. Note that we drop the subscript of $L$ in (7) to generally represent the LLRs in (5) and 
(6). To generate the EXIT band chart, the PDFs of the LLRs corresponding to $I_{L_{M, a}^{[s]}}$ and $I_{L_{D, a}^{[s]}}$ are assumed to be Gaussian distributed. When $I_{L_{M, e}^{[s]}}^{[s]}$ and $I_{L_{D, e}}^{[s]}$ are calculated, the PDFs of $L_{M, e}^{[s]}$ and $L_{D, e}^{[s]}$ are obtained by the histogram method [11].

To obtain the EXIT transfer characteristic of the MSDD SISOD, we repeatedly perform open loop simulations by changing the channel realization seed [s] and the mutual information of the input priori information $I_{L_{M, a}^{[s]}}$ at the same SNR. For the considered systems with finite-length LDPC codes, we obtain various values of $I_{L_{M, e}^{[s]}}$ for different channel realizations even with the same values of $I_{L_{M, a}^{[s]}}$ and SNR. Hence, in contrast to the considered systems with infinite-length LDPC codes, whose EXIT transfer characteristic of MSDD SISOD is a single curve, for the considered systems with finite-length LDPC codes, the EXIT transfer characteristic of the MSDD SISOD is a band of curves of $I_{L_{M, e}^{[s]}}$ with respect to $I_{L_{M, a}^{[s]}}$. Similarly, the transfer characteristic of the LDPC decoder for finite-length LDPC codes is also a band of curves of $I_{L_{D, e}^{[s]}}$ with respect to $I_{L_{D, a}^{[s]}}$. Similar to the analysis of [12], the EXIT band of the MSDD SISOD (LDPC decoder) can be represented using the average curves $\operatorname{avg}\left(I_{L_{M, e}^{[s]}}\right)\left(\operatorname{avg}\left(I_{L_{D, e}^{[s]}}\right)\right)$, and the upper and lower bound $\operatorname{avg}\left(I_{L_{M, e}^{[s]}}\right) \pm \operatorname{std}\left(I_{L_{M, e}^{[s]}}\right)\left(\operatorname{avg}\left(I_{L_{D, e}^{[s]}}\right) \pm \operatorname{std}\left(I_{L_{D, e}^{[s]}}\right)\right.$, respectively), where $\operatorname{avg}(\cdot)$ and $\operatorname{std}(\cdot)$ represent the average and the standard deviation, respectively. The EXIT band chart can be obtained by plotting the EXIT bands of the MSDD SISOD and the LDPC decoder into a signal diagram by switching the $x$-axis and the $y$-axis.

Figure 2 shows the EXIT band charts of the considered systems with rate-1/2 $(3,6)$ regular LDPC codes with different finite code lengths over AWGN channels with BPSK, where the number of inner iterations of the LDPC decoder is 10. For comparison, the EXIT band chart for a very long code length of 100800 (can be viewed as infinitelength) is also presented. We can observe that the shorter the code length is, the wider the EXIT bands become. When the code length is increased to 100800 , the widths of the EXIT bands become zero. That is, the EXIT band chart of infinite-length codes is actually equivalent to the ordinary EXIT chart. For infinite-length LDPC codes, it is known that each frame has similar asymptotic performance when the SNR is larger than the asymptotic decoding threshold; thus the uncorrectable frames whose performance can only be improved marginally by increasing the iteration number appear only at low SNRs (those SNRs smaller than the asymptotic decoding threshold). Whereas for the same LDPC code ensemble with finite-length, due to the overlap of the EXIT curve bands, uncorrectable frames still exist at certain SNRs which are above the asymptotic decoding threshold. This means that uncorrectable frames appear at low SNRs and also at medium SNRs. This conclusion can be further supported by Fig. 3, which shows three typical simulated snapshot iterative decoding trajectories for the considered systems with rate- $1 / 2(3,6)$ regular LDPC codes of length 1008 and $S=4$ at $\mathrm{SNR}=3.8 \mathrm{~dB}$. It is shown that
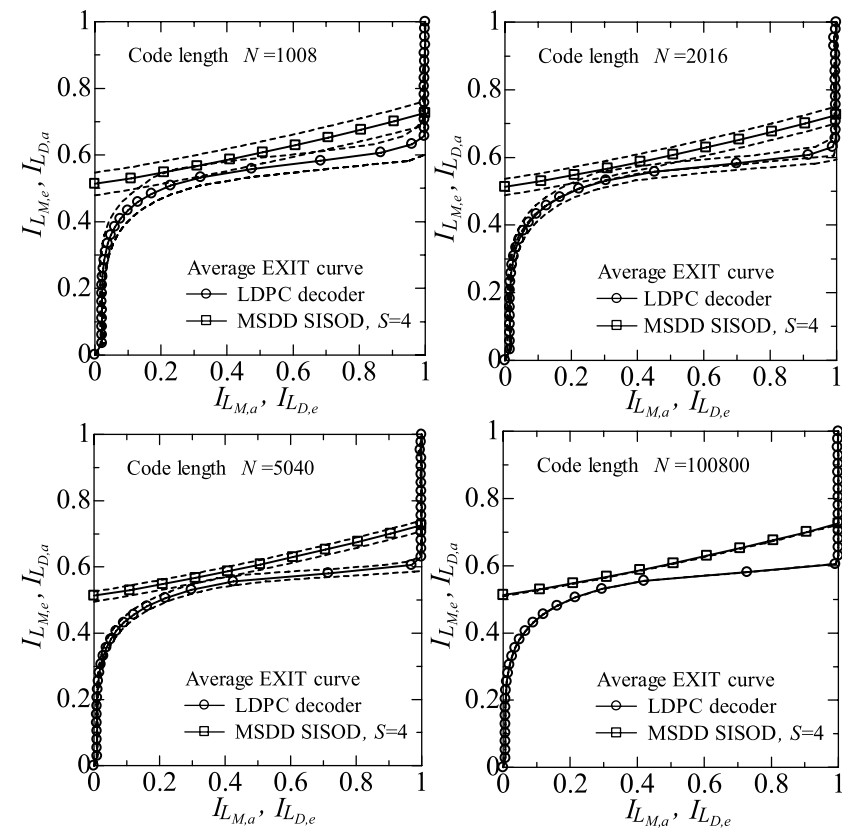

Fig. 2 EXIT band charts of the considered systems for MSDD SISOD with $S=4$ and rate-1/2 $(3,6)$ regular LDPC codes with different code lengths over AWGN channels with BPSK at $\mathrm{SNR}=3.5 \mathrm{~dB}$; inner iteration number of the LDPC decoder is 10; repeated 10000 frames.

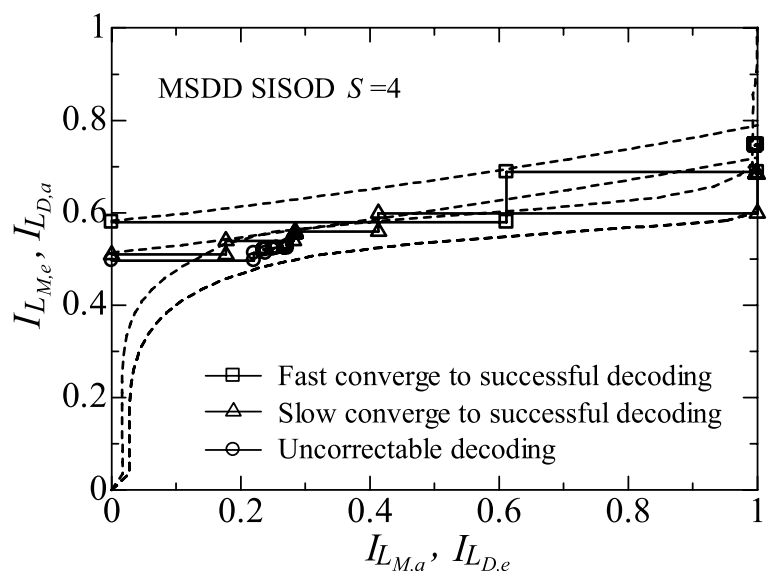

Fig. 3 Typical simulated snapshot iterative decoding trajectories of the considered systems for MSDD SISOD with $S=4$ and rate-1/2 $(3,6)$ regular LDPC codes with length 1008 over AWGN channels with BPSK at SNR = $3.8 \mathrm{~dB}$; inner iteration number of the LDPC decoder is 10 .

these iterative decoding trajectories exhibit a greater variation from frame to frame when the code length is short. Some frames quickly or slowly converge to successful decoding, while some frames cannot converge to successful decoding.

Next, we analyze the impact of the OWS of the MSDD SISOD on system performance. Figure 4 shows the average EXIT curves of the LDPC decoder and the MSDD SISOD with different OWS at SNR $=3.5 \mathrm{~dB}$. It is shown that the slopes of the EXIT curves of the MSDD SISOD increase with an increase in $S$, which implies that the performance of the systems can be improved by increasing $S$. How- 


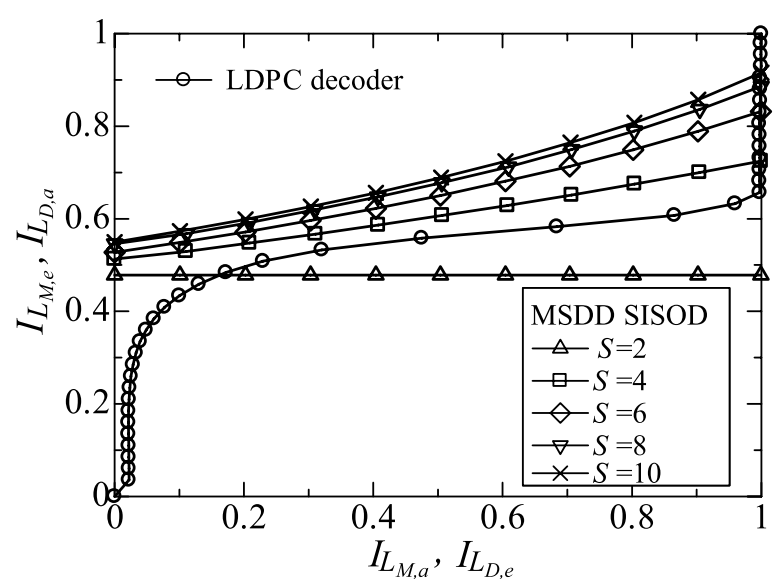

Fig. 4 Average EXIT curves of the considered systems for MSDD SISOD with different $S$; rate-1/2 $(3,6)$ regular LDPC codes with length 1008 over AWGN channels with BPSK at SNR $=3.5 \mathrm{~dB}$; inner iteration number of the LDPC decoder is 10; averaged over 10000 frames.

ever, at low SNRs, e.g. $1.0 \mathrm{~dB}$, it can be expected that the EXIT band of the MSDD SISOD is almost entirely under the EXIT band of the LDPC decoder even with large values of $S$, which means that increasing $S$ at low SNRs cannot improve the system performance much. On the other hand, at high SNRs, e.g. $5.0 \mathrm{~dB}$, it can be expected that a large tunnel is opened between the EXIT bands of the MSDD SISOD and the LDPC decoder even for $S=2$, which means that most of the frames can be decoded successfully using a small value of $S$. While at medium SNRs, as shown in Fig. 2 for $N=1008$ at $\mathrm{SNR}=3.5 \mathrm{~dB}$, it can be expected that the fraction of the overlap between the two EXIT bands will decrease with an increase in $S$, and a tunnel will be opened when $S \geqslant 6$. This means that the probability of successful decoding can be increased, and thus a significant improvement in average bit error rate (BER) performance can be achieved by increasing $S$ at medium SNRs.

\section{AIDA}

\subsection{Motivation of AIDA}

The OWS of the MSDD SISOD and the outer iteration number are the two key parameters that determine the iterative decoding complexity and delay of the considered systems. It is known that the complexity of MSDD grows exponentially with the OWS. Therefore, the complexity of the MSDD SISOD will become prohibitively high as the OWS becomes large. Especially for iterative decoding systems with large outer iteration numbers, this high complexity will result in an unacceptable decoding delay, which makes it difficult to achieve a realistic system.

In [7], an approach which gradually increases the OWS in accordance with the iteration number of turbo coded systems with MSDD by looking up an OWS table, was proposed based on the analysis of the iterative decoding process using the EXIT chart. It is proved that this approach can reduce the complexity of the iterative MSDD decoding with negligible performance loss compared with the OWS with a fixed large value [7]-[9]. In this approach, the OWS table and the maximum outer iteration number are designed based on a designed iterative decoding trajectory obtained from the EXIT chart. Since the two parameters are designed for a target BER at the expected SNR, the decoding complexity and delay cannot be significantly reduced at all SNRs by using this approach. Moreover, the design of the two parameters is based on the assumption that the iterative decoding trajectory is well matched to the EXIT functions of the two component decoders of the iterative decoder, which is valid for codes with very long length, but not for codes with short length.

Based on the analysis results of Sect. 2.3, a small OWS and a small outer iteration number are sufficient for low SNRs and high SNRs. Furthermore, for medium SNRs, the performance improvement achieved by increasing the OWS and the outer iteration number varies from frame to frame when the code length is finite. Therefore, it is necessary to propose an adaptive iterative decoding approach (AIDA) to adjust the OWS and the outer iteration number adaptively to reduce the iterative decoding complexity and delay of the considered systems with finite-length LDPC codes in a changing SNR environment.

\subsection{Principle of AIDA}

In the proposed AIDA, in contrast to adjusting the OWS of the MSDD SISOD and the outer iteration number according to the predesigned OWS table and the maximum outer iteration number as in [7], the two parameters are adjusted according to the convergence status of the iterative decoding. Figure 5 shows the structure of AIDA. Let $S_{i}$ and $S_{\text {max }}$ denote the OWS at the $i$ th iteration and the predefined maximum OWS of the MSDD SISOD, respectively. In the first iteration, $S_{1}$ is set to 2 . If the decoding by the LDPC decoder is successful, the iterative decoding will be stopped automatically; otherwise, the SC will be checked. If the evaluation of the $\mathrm{SC}$ indicates that the requirement of stopping iteration is satisfied, the iterative decoding will be stopped; otherwise, the iterative decoding will continue to be executed, and the OWS will be increased by a predefined fixed value $\Delta S$ in the next iteration if $S_{i}<S_{\max }$. This process is repeated until the requirement of stopping iteration is satisfied, or until the predefined maximum iteration number is reached or decoding is successful. In the SC, the requirement of stopping iteration is an indicator which can reflect the convergence of the iterative decoding.

The SC is the key part in AIDA. In order for AIDA to be able to reduce the iterative decoding complexity and delay with very little performance loss, a well designed SC should be able to timely and accurately judge the convergence of the iterative decoding. Up to now, many SCs have been proposed to reduce the iteration number for turbo coded systems. In the following, some representative existing SCs are briefly introduced, and these criteria for the considered systems are studied in Sect. 3.3. Then, to circum- 


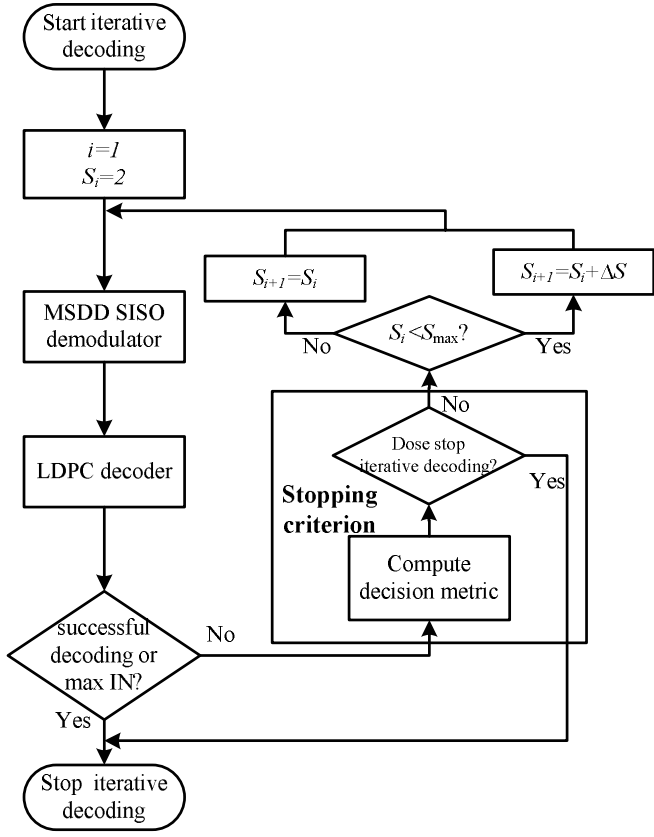

Fig. 5 Structure of AIDA, where IN is the acronym of iteration number.

vent the disadvantages of these criteria for the considered systems, a new SC is proposed in Sect. 3.4.

\subsection{Existing SCs for the Considered Systems}

Let $L_{e 1}\left(c_{k}\right)$ and $L_{e 2}\left(c_{k}\right)$ denote the extrinsic LLRs of the $k$ th code bit of the two component decoders of the turbo decoder, respectively, and let $N$ denote the length of turbo codes. The existing SCs are introduced as follows.

1) $\mathrm{CE}$ criterion: $\mathrm{CE}$ is used to measure the closeness of two distributions. At the ith iteration, let $p_{1}^{i}\left(c_{k}\right)$ and $p_{2}^{i}\left(c_{k}\right)$ denote the a posteriori probability distributions of the outputs of the two component decoders of the turbo decoder, respectively. The CE between $p_{1}^{i}\left(c_{k}\right)$ and $p_{2}^{i}\left(c_{k}\right)$ can be expressed as

$$
T(i)=E\left\{\log \frac{p_{2}^{i}\left(c_{k}\right)}{p_{1}^{i}\left(c_{k}\right)}\right\} \approx \sum_{k=1}^{N} \frac{\left|L_{e 2}^{i}\left(c_{k}\right)-L_{e 2}^{i-1}\left(c_{k}\right)\right|^{2}}{\exp \left(L_{e 1}^{i}\left(c_{k}\right)\right)} .
$$

With an increase of the iteration number, the CE becomes smaller and smaller. When $T(i)$ is smaller than a predefined threshold $\left(10^{-2} \sim 10^{-4}\right) T(1)$, the iterative decoding is terminated.

2) SCR criterion: SCR is based on measuring the sign changes $C(i)$ of $L_{e 2}\left(c_{k}\right)$ from iteration (i-1) to iteration $i$. The principle of SCR is to compute $C(i)$ at each iteration, and the iterative decoding is terminated when $C(i)$ is smaller than the threshold $(0.005 \sim 0.03) N$.

3) SDR criterion: Let $D(i)$ denote the number of sign differences between $L_{e 1}\left(c_{k}\right)$ and $L_{e 2}\left(c_{k}\right)$ in the same iteration. The principle of SDR is to compute $D(i)$ after each iteration and to terminate the iterative decoding when $D(i)$ is smaller than the threshold $(0.001 \sim 0.01) N$.
4) ME criterion: This approach is based on monitoring $M_{|L|}$, the mean of absolute LLR values of the second decoder over a block after each iteration. Simulation shows that $M_{|L|}$ increases as the number of errors decrease. Therefore, the iterative decoding is terminated when $M_{|L|}$ is bigger than a predifined threshold.

Since the iterative decoder of the considered systems, which consists of a demodulator and a LDPC decoder, is different from the turbo decoder, the assumptions which are used to obtain the approximation of $\mathrm{CE}$ as expressed in (8) [14], do not hold for the iterative decoder of the considered systems. Thus, the CE criterion is modified to be used only for the LDPC decoder, and the decision metric of the CE criterion for the considered systems is turned into

$$
T(i) \approx \sum_{k=1}^{N} \frac{\left|L_{D, p}^{i}\left(c_{k}\right)-L_{D, p}^{i-1}\left(c_{k}\right)\right|^{2}}{\exp \left(L_{D, p}^{i-1}\left(c_{k}\right)\right)} .
$$

That is, the CE for the considered systems is used to measure the closeness of the a posteriori probability outputted from the LDPC decoder in two consecutive outer iterations. For SCR, SDR and ME criteria, these SCs can be used in the considered systems directly. These SCs have been proved to be the efficient approaches to detect the successful decoding before reaching the maximum iteration number by comparing a predefined threshold. However, compared with turbo codes, LDPC codes can detect successful decoding to stop the iteration by checking the parity check constraints of LDPC codes. Thus, the SC for the considered systems is more concerned with stopping the uncorrectable decodings which usually occur at low to medium SNRs for our considered systems. In this situation, the stopping rules and the thresholds of the SCs mentioned above should be redesigned to be applicable for this purpose.

To understand how to design the stopping rules and the thresholds of these SCs for the considered systems, the average evaluations of the decision metrics of these SCs variation with SNR and outer iteration number are shown in Fig. 6. We can observe that the stopping rules of the CE, SCR and ME criteria for our target are that the evaluation of their decision metrics should be smaller than a threshold. On the contrary, the stopping rule of the SDR criterion is that the evaluation of its decision metric should be bigger than a threshold. However, we can observe that they do not work well at all SNRs based on the redesigned stopping rules. For CE and SCR criteria, the iteration may be stopped prematurely at medium and high SNRs, which results in performance losses at these SNRs, since the evaluations of their decision metrics vary non-monotonically with SNR as shown in Figs. 6(a) and (b). On the other hand, for SDR and ME criteria, it is possible to design proper thresholds for them at low SNRs based on Figs. 6(c) and (d). The low SNR region can be determined with the aid of the asymptotic decoding threshold predicated by the EXIT chart for the considered systems. In this SNR region, the iterative decoding of each frame should be stopped after the first or second iteration also for finite-length LDPC codes. However, it 


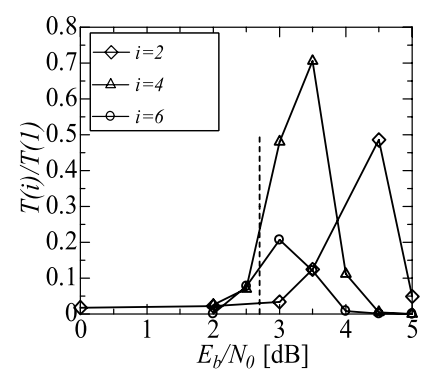

(a) $\mathrm{CE}$

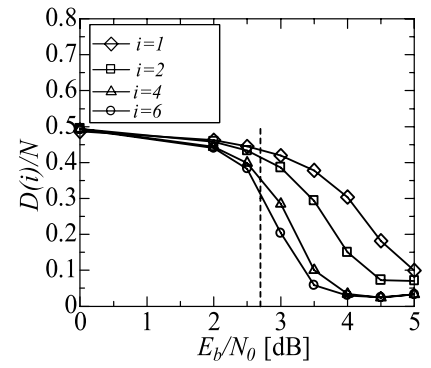

(c) SDR

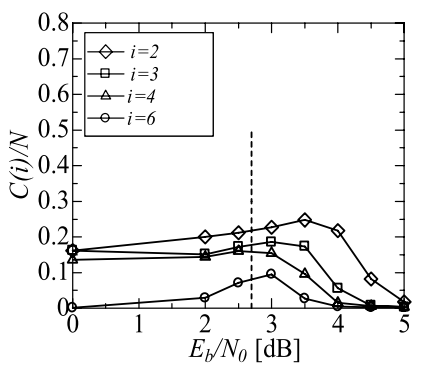

(b) SCR

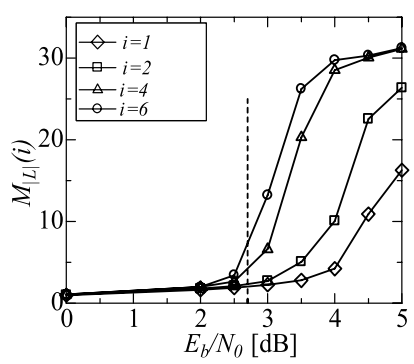

(d) $\mathrm{ME}$
Fig. 6 Average evaluations of the decision metrics of the existing SCs variation with SNR and outer iteration number; rate-1/2 $(3,6)$ regular LDPC codes with length 1008 over AWGN channels with BPSK; OWS of MSDD SISOD corresponding to the outer iteration number from 1 to 6 is $[2,4,6,8,8,8]$; inner iteration number of the LDPC decoder is 10 ; averaged over 10000 frames.

is difficult to design proper thresholds of them also valid for medium SNRs, since the iterative decoding characteristics greatly vary from frame to frame for the considered systems at medium SNRs as discussed in Sect. 2.3. Moreover, the low SNR region where is no need for increasing iteration number is changed with a change in the LDPC code and transmission channel parameters, which makes the defined thresholds of these SCs need to be redesigned again.

\subsection{Proposed SC}

The basic reason of the disadvantages of these SCs is that these approaches are not good methods to track the convergence status of the iterative decoding. From the decoding trajectories of Fig. 3, we can observe that the value of $I_{L_{D, e}}$ increases significantly between two consecutive outer iterations if the iterative decoding can improve the system performance effectively, whereas the value of $I_{L_{D, e}}$ remains almost unchanged as the number of iterations increases when the iterative decoding has converged. Thus, the change of $I_{L_{D, e}}$ between two consecutive outer iterations can reflect the convergence status of the iterative decoding. These observations motivate us to propose a new SC named DMI criterion, which is based on tracking the difference of the output mutual information of the LDPC decoder between two consecutive outer iterations. Based on this idea, at the $i$ th iteration, the decision metric of the DMI criterion can be written as

$$
\Delta I_{L_{D, e}}^{i}=I_{L_{D, e}}^{i}-I_{L_{D, e}}^{i-1} .
$$

If $\Delta I_{L_{D, e}}$ is smaller than a threshold $T h$, which means that

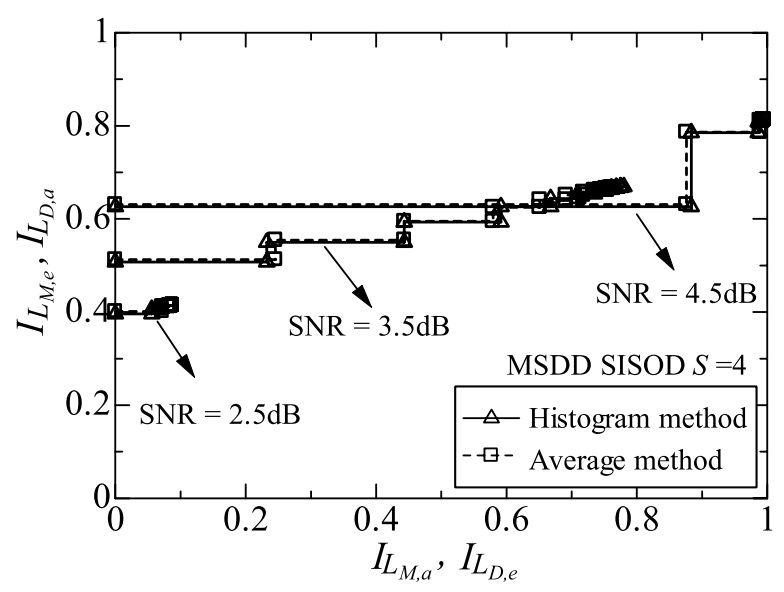

Fig. 7 Averaged simulated iterative decoding trajectories of the considered systems evaluated by different approaches; MSDD SISOD with $S=4$ and rate-1/2 $(3,6)$ regular LDPC codes with length 1008 over AWGN channels with BPSK at different SNRs; inner iteration number of the LDPC decoder is 10; averaged over 10000 frames.

the iterative decoding has converged, the iterative decoding is stopped; otherwise, the next iteration is executed.

The computation of $I_{L_{D, e}}$ using (7), also called the histogram method, needs the information about the transmitted coded bits and the PDF of LLR values, which is impractical for realistic systems where the transmitted coded bits are unknown at the receiver. Actually, (7) can be computed by the average method shown below without these requirements [20]

$$
I_{L} \approx 1-\frac{1}{N} \sum_{n=1}^{N} H_{b}\left(\frac{e^{-\left|l_{n}\right| / 2}}{e^{\left|l_{n}\right| / 2}+e^{-\left|l_{n}\right| / 2}}\right),
$$

where $N$ is the code sequence length, $l_{n}$ is the LLR value of the $n$th bit of the code sequence, and $H_{b}$ is the binary entropy function

$$
H_{b}(p)=-p \log _{2} p-(1-p) \log _{2}(1-p), 0 \leqslant p \leqslant 1 .
$$

Equation (11) is valid when the APP decoder is employed even if the distribution of the LLR outputs of the decoder is non-Gaussian or unknown distributions [20]. Figure 7 shows the comparison of the averaged simulated iterative decoding trajectories of the considered systems at different SNRs obtained by the histogram method and the average method, respectively. It is shown that the average method is a good approximation for the histogram method. Therefore, it is reasonable to use (11) to compute $I_{L_{D, e}}$ after each outer iteration for the considered systems where the LDPC decoder uses the sum-product algorithm.

We must point out that the idea of SC based on (10) has been proposed in [18] for turbo HARQ schemes, which is used to reduce the complexity while maintaining a high throughput and a low packet-loss-ratio. However, since the purpose of our work is different from that of [18], the SC based only on (10) is not the most effective approach for our considered systems, which will be explained below and 
be supported in Sect. 4.2. Based on this fact, the principle of our proposed DMI criterion, which is also the difference between our proposed DMI criterion and the SC of [18], is explained from the following two aspects.

On one hand, it is obvious that using the SC with only the decision metric (10), the system will execute at least two iterations before successful decoding. However, in fact, in most cases, iterative decoding only needs to be executed once at low SNRs. From Figs. 2 and 4, we can also observe that the EXIT curves of the MSDD SISOD and the LDPC decoder intersect on the very left side of the EXIT band chart at low SNRs corresponding to very small value of $I_{L_{D, e}}$. Thus, to further reduce unnecessary iterations at low SNRs, before evaluating the decision metric of the DMI criterion, $I_{L_{D, e}}^{i}$ is compared with another threshold $T h_{L}$ to decide whether to stop the iterative decoding immediately.

On the other hand, as shown in Fig. 3, at medium SNRs, the iterative decoding of some frames corresponding to the typical iterative decoding behavior of slow convergence has the following features: $I_{L_{D, e}}^{i}$ increases to a relatively large value and seems to be unchanged (that is, $\Delta I_{L_{D, e}}^{i}$ very small) after the first few iterations, but $I_{L_{D, e}}^{i}$ can increase to the value of the right side of the EXIT band chart after some iterations, which results in successful decoding at last. Owing to this fact, if the SC only considers (10), this type of decoding behavior will be prematurely stopped, resulting in a performance loss. Therefore, The proposed SC should consider how to avoid prematurely stopping this type of decoding behavior. We solve this problem as follows. We define a threshold $T h_{M}$ which is bigger than the threshold $T h_{L}$. At the $i$ th iteration, if $\Delta I_{L_{D, e}}^{i}<T h$ but $I_{L_{D, e}}^{i}>T h_{M}$, which means that the current iterative decoding may be the type of the slow convergence, the iterative decoding is not stopped immediately and let an indicator $S N=S N+1$. Here $S N$ is used to represent the number of times that $\Delta I_{L_{D, e}}^{i}<T h$ when $I_{L_{D e}}^{i}>T h_{M}$ during the current iterations. If $S N$ is bigger than a defined number of times $T h_{N}$, which means that the current iterative decoding is an uncorrectable decoding with high probability, then decoding is stopped; otherwise, the current iterative decoding may be the type of the slow convergence decoding, thus go to the next iteration.

The proposed DMI criterion is summarized as follows:

At the $i$ th outer iterative decoding $(S N=0)$ :

Step 1: Compute $I_{L_{D, e}}^{i}$ using equation (11) at the $i$ th iteration.

Step 2: If $I_{L_{D, e}}^{i}<T h_{L}$, stop the iterative decoding; otherwise, go to step 3 .

Step 3: If $I_{L_{D e}}^{i}<T h_{M}$ and $\Delta I_{L_{D e}}^{i}<T h$, stop the iterative decoding; otherwise, go to step 4 .

Step 4: If $I_{L_{D, e}}^{i}>T h_{M}$ and $\Delta I_{L_{D, e}}^{i}<T h$, let $S N=$ $S N+1$. If $S N>T h_{N}$, stop the iterative decoding; otherwise, execute the next iteration.

Actually, we can find that the SC of [18] is equivalent to the proposed DMI criterion with $T h_{L}=0.0$ and $T h_{M}=$ 1.0. It should be emphasized that the performance of AIDA with the DMI criterion depends on the values of the thresholds $T h, T h_{L}, T h_{M}$ and $T h_{N}$. The EXIT band chart of the system can simplify the process of choosing of these thresholds. Although, generally speaking, the best choice of these thresholds should be optimized for particular system parameters, the performance of the DMI criterion with a set of determined thresholds is robust when the LDPC code and transmission channel environment parameters are changed, which will be supported by the simulation results presented in the next section.

\section{Simulation Results and Analysis}

The performances of the proposed AIDA with the existing SCs and the proposed SC for the considered systems are evaluated and analyzed using computer simulations. Unless otherwise indicated, the following simulation parameters are used for our simulations. We consider rate-1/2 $(3,6)$ regular LDPC codes with length 1008 over AWGN channels. The coded bits are modulated using BPSK for simplicity. The maximum number of outer iterations between the MSDD SISOD and the LDPC decoder is set to 6. The maximum number of inner iterations of the LDPC decoder is set to 10. In AIDA, $S_{\max }$ and $\Delta S$ are set to 8 and 2 , respectively.

\subsection{Selection of the DMI Criterion Thresholds}

Based on the EXIT band chart analysis of the considered systems with the above simulation parameters, which is similar to the analysis of Figs. 2, 3, 4 and 7, the approximate ranges of the thresholds $T h, T h_{L}, T h_{M}$ and $T h_{N}$ can be first determined as follows: $T h<0.03, T h_{L}<0.12$, $0.3<T h_{M}<0.7$ and $T h_{N}<3$. Then, we can select appropriate values for them in their corresponding ranges. More specifically, we first evaluate the variation of the average number of outer iterations and the BER performance of the considered systems using AIDA with the DMI criterion with different values of threshold $T h$ as shown in Fig. $8 . T h_{L}$ and $T h_{M}$ are set to $0.0,1.0$, respectively. In this situation, the performance of the DMI criterion is determined only by the threshold $T h$. It is shown that the smaller $T h$ is, the smaller performance loss is, but the larger number of outer iterations is required. In order to make a good trade-off between the iterative decoding complexity and the performance loss, the value of $T h$ that is selected is 0.02 . After determining the value of $T h$, we can then determine the value of $T h_{L}$, and then determine the values of $T h_{M}$ and $T h_{N}$ together at last using simulations similar to the process of the selection of $T h$. For the sake of conciseness, the specific processes used in the selection of $T h_{L}, T h_{M}$ and $T h_{N}$ are not presented in detail here. Finally, the thresholds $T h, T h_{L}, T h_{M}$ and $T h_{N}$ of the proposed DMI criterion are set to $0.02,0.07,0.5$ and 1 , respectively, for the following simulations. 


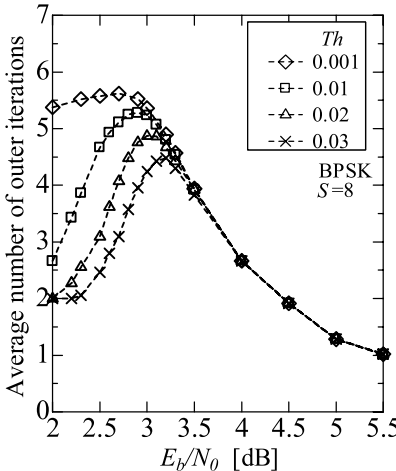

(a)

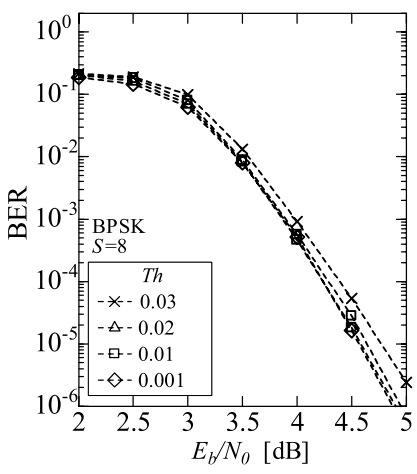

(b)
Fig. 8 Performance of AIDA using the DMI criterion variation with the value of threshold $T h ; T h_{L}=0.0$ and $T h_{M}=1.0$; rate-1/2 $(3,6)$ regular LDPC codes with length 1008 over AWGN channels with BPSK. (a) Average number of outer iterations. (b) BER performance.

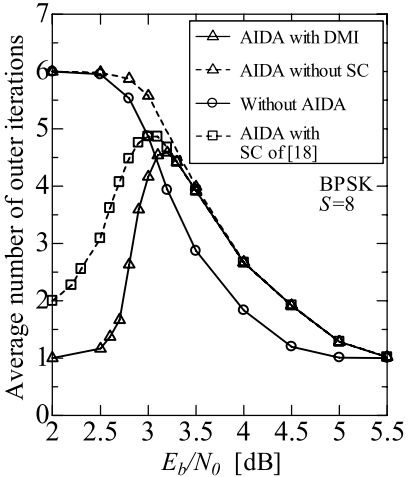

(a)

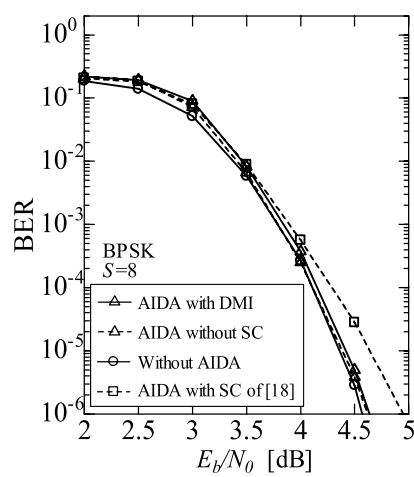

(b)
Fig.9 Performance of the considered DE-LDPC coded systems with and without AIDA; rate-1/2 $(3,6)$ regular LDPC codes with length 1008 over AWGN channels with BPSK. (a) Average number of outer iterations. (b) BER performance.

\subsection{Performance of AIDA with the DMI Criterion}

Figure 9(a) shows the average number of outer iterations of the considered systems using AIDA without SC and with SC of the proposed DMI criterion. Here, AIDA without SC is that the early stopping approach is not used in AIDA. In case of AIDA without SC, the OWS of MSDD SISO corresponding to the outer iteration number from 1 to 6 is $[2,4,6,8,8,8]$, and the outer iterations are stopped when the maximum outer iteration number is reached or a legitimate codeword is found. The simulation result of the considered systems with fixed $S=8$ without AIDA is also presented for comparison. Since the LDPC decoder can detect successful decoding and stop the iteration by checking the parity check constraints of LDPC codes, it is shown that the average number of outer iterations required by AIDA without $\mathrm{SC}$ is gradually decreased with an increase in SNR. When the DMI criterion is used in AIDA, since the DMI criterion adjusts the number of outer iterations based on the convergence status of the outer iterations, we observe that the av-

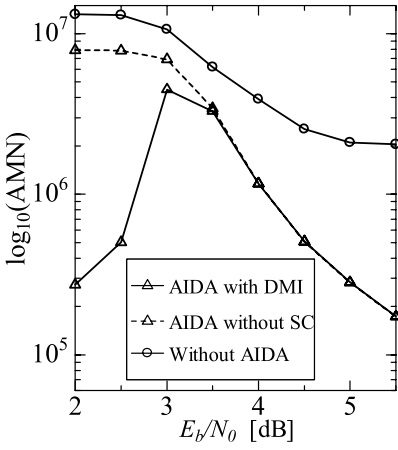

(a)

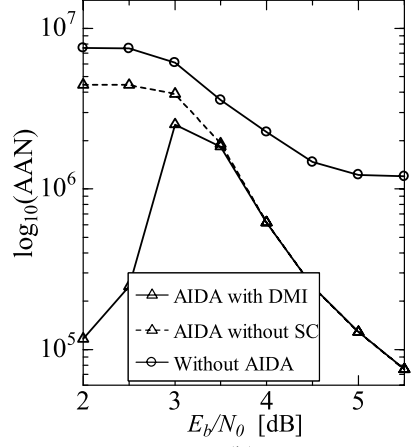

(b)
Fig. 10 Computational complexity of the iterative decoding of each frame of the considered DE-LDPC coded systems with and without AIDA; rate-1/2 $(3,6)$ regular LDPC codes with length 1008 over AWGN channels with BPSK. (a) AMN. (b) AAN.

erage number of outer iterations can be reduced efficiently by AIDA with the DMI criterion at low and medium SNRs. More specifically, the outer iterations are stopped as early as possible at low SNRs, where the performance cannot be improved by increasing the OWS and the number of outer iterations. At medium SNRs, where the performance improvement can be gradually achieved by increasing the OWS and the number of outer iterations, the average iteration number is gradually increased. While at high SNRs, the average numbers of outer iterations required by AIDA with and without the DMI criterion are the same, since almost all of the frames can be successfully decoded in the first few iterations.

Figure 9 also shows the performance comparison between AIDA with the DMI criterion and AIDA with the SC of [18] that is equivalent to setting $T h=0.02, T h_{L}=0.0$ and $T h_{M}=1.0$ for the DMI criterion. We can observe that for the systems considered here, the proposed DMI criterion performs better than the SC of [18] in terms of the required average number of outer iterations at low SNRs and the performance loss at medium and high SNRs, which supports the discussion of the difference between the proposed DMI criterion and the SC of [18] in Sect. 3.4.

Next, we analyze the reduction of the iterative decoding complexity by using AIDA. The evaluation of the iterative decoding complexity needs to consider the number of outer iterations and the complexity of the MSDD SISOD and the LDPC decoder. Since the LDPC decoder can detect successful decoding and automatically stop the iteration, we evaluate the iterative decoding complexity by mainly considering the average multiplication number (AMN) and the average addition number (AAN) of the iterative decoding of each frame for the considered systems with and without AIDA as shown in Fig. 10. The method introduced in [21] is used to evaluate the decoding complexity of the LDPC decoder using the sum-product algorithm. It should be noted that since the number of divisions is far less than the number of multiplications in each iteration, it is not considered in the evaluation of the iterative decoding complexity. In addition, 


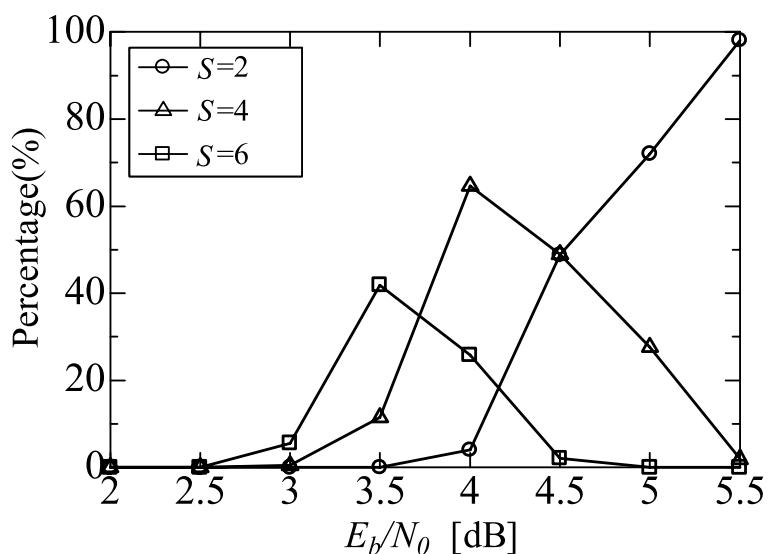

Fig. 11 Percentage of successful decodings of the considered DE-LDPC coded systems with $S<8$ when AIDA is used; rate-1/2 $(3,6)$ regular LDPC codes with length 1008 over AWGN channels with BPSK.

the calculation of the logarithmic term in (3) also is ignored.

Although from Fig. 9 it can be seen that the average numbers of outer iterations from $2.7 \mathrm{~dB}$ to $5.25 \mathrm{~dB}$ required by AIDA without SC are about 0.2 to 0.5 times more than that required without using AIDA for the considered systems, Fig. 10 shows that both the AMN and AAN are significantly reduced by AIDA without the SC at all SNRs. For example, for SNR $=4.5 \mathrm{~dB}$, about $20 \%$ of the complexity of the iterative decoding without AIDA is required by using AIDA. This is because the fraction of successful decoding achieved by the MSDD SISOD with small $S$ increases with an increase in SNR values as shown in Fig. 11. From Fig. 10, we also observe that AIDA using the DMI criterion can further reduce the decoding complexity at low and medium SNRs. For example, only about $3 \%$ of the complexity of the iterative decoding without AIDA is required by using AIDA with the DMI criterion. Combining the above observations and the results of the BER performance comparison of the considered systems with and without AIDA as shown in Fig. 9(b), we can conclude that the proposed AIDA with the DMI criterion can significantly reduce the iterative decoding complexity and delay with negligible performance loss.

\subsection{Performance of AIDA with different SCs}

In the following, performances of AIDA with different SCs are compared and analyzed. The principle of the selection of the thresholds of the aforementioned existing SCs, which is the same as that of [17], is that the outer iteration should be stopped immediately when the SNR is below the asymptotic decoding threshold of the considered systems. It should be noted that at least two outer iterations are performed before successful decoding when CE and SCR are used. Based on the discussion of Sect. 3.3, according to Fig. 6 and the asymptotic decoding threshold for the considered systems with $S=8$ and the rate-1/2 $(3,6)$ regular LDPC code ensemble over AWGN channels with BPSK as shown by the dotted lines in Fig. 6, the approximate ranges of their thresh-

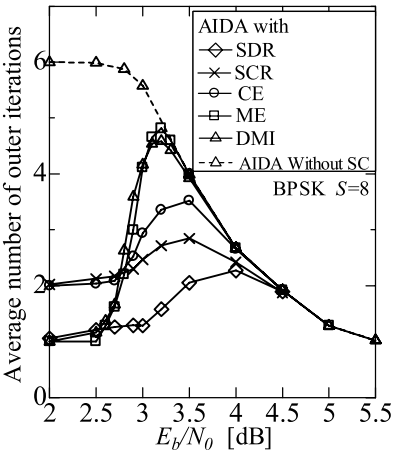

(a)

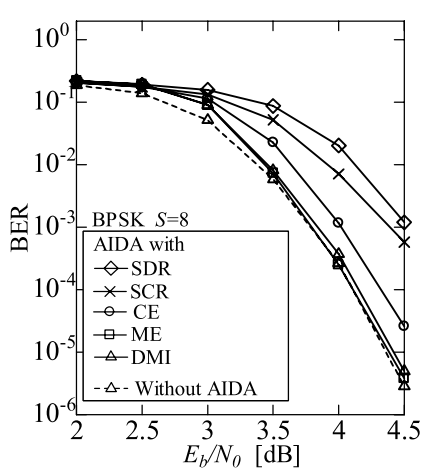

(b)
Fig. 12 Performance of AIDA with different SCs for the considered DELDPC coded systems with rate-1/2 $(3,6)$ regular LDPC codes with length 1008 over AWGN channels with BPSK. (a) Average number of outer iterations. (b) BER performance.

olds can be determined from the $y$-axes of Fig. 6. Then, the preferred values of their thresholds can be selected to meet the above principle in their corresponding ranges using simulations similar to Fig. 8, the detailed process of which is omitted for the sake of conciseness. For the systems considered here, the thresholds of CE, SCR, SDR and ME criteria are selected as $0.035,0.24,0.37$ and 2.2 , respectively.

The same simulations of Fig. 9 are tested for AIDA with different SCs, as shown in Fig. 12. For AIDA with CE, SCR and SDR, it is shown that the average numbers of outer iterations are significantly reduced with very little performance loss at low SNRs. However, large BER performance losses are caused at medium and high SNRs, since some correctable decodings are prematurely stopped by the three criteria. This implies that the three existing SCs are not well suited for stopping the iteration of the uncorrectable decodings for our considered systems. On the other hand, for AIDA using the ME criterion, we observe that it has similar performance to AIDA using the proposed DMI criterion.

To further evaluate and compare the performance of the ME criterion and the DMI criterion for AIDA, the same simulations of Fig. 12 are also tested for different code structures and transmission channel environments respectively while keeping other simulation parameters constant. Figure 13 shows the simulation results for rate- $3 / 4(3,12)$ regular LDPC codes with length 1008 over AWGN channels. Figure 14 shows the simulation results for rate- $1 / 2(3,6)$ regular LDPC codes with length 1008 over non-frequency selective Rayleigh fading channels with normalized maximum Doppler frequency $f_{D} T_{s}=0.01$. It is shown that the ME criterion with the previously defined threshold 2.2 for the two cases is not as effective as it is for rate-1/2 $(3,6)$ regular LDPC codes over AWGN channels in term of the performance of the reduction of the average number of outer iterations. These observations suggest that the performance of the existing ME criterion is easily affected by a change in the LDPC code and transmission channel parameters, and thus the threshold needs to be redesigned for the new conditions, 


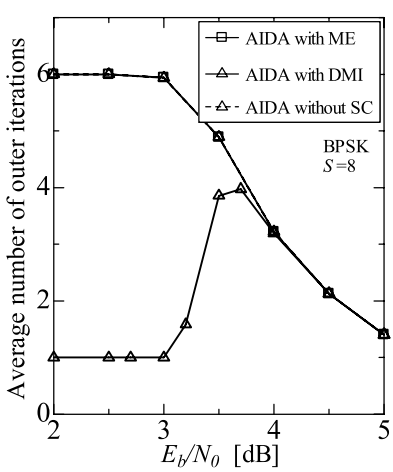

(a)

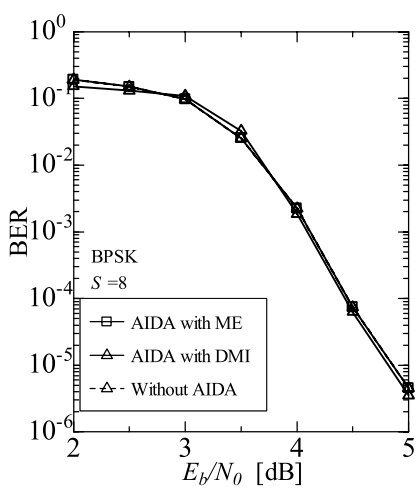

(b)
Fig. 13 Performance of the considered DE-LDPC coded systems with and without AIDA for rate-3/4 $(3,12)$ regular LDPC codes with length 1008 over AWGN channels with BPSK. (a) Average number of outer iterations. (b) BER performance.

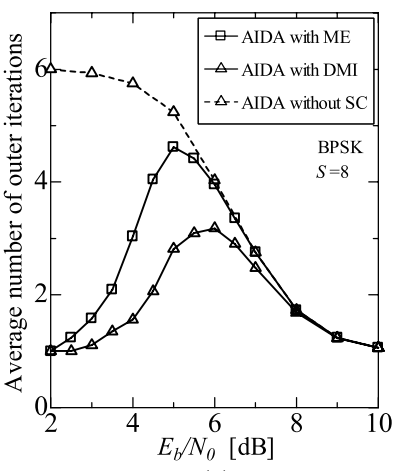

(a)

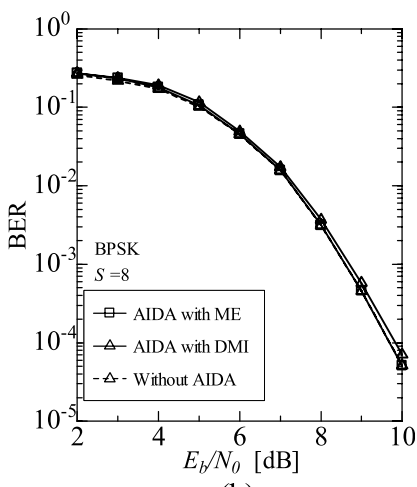

(b)
Fig. 14 Performance of the considered DE-LDPC coded systems with and without AIDA for rate-1/2 $(3,6)$ regular LDPC codes with length 1008 over non-frequency selective Rayleigh fading channels with normalized maximum Doppler frequency $f_{D} T_{s}=0.01$ and BPSK. (a) Average number of outer iterations. (b) BER performance.

which supports the discussion in Sect. 3.3. On the contrary, we can find that even if these conditions are changed, the proposed DMI criterion with previously defined thresholds can still effectively reduce the average number of outer iterations with very little performance degradation at all SNRs, which means that the proposed DMI criterion is more robust than the ME criterion. This is due to the fact that the proposed DMI criterion stops the iteration based on the convergence status of the iterative decoding rather than comparing a predefined threshold for a certain SNR region like the existing SCs.

In addition, although the threshold of the ME criterion can be redesigned to suit new conditions, the workload of this process is large, since its threshold needs to be carefully designed based on a large number of simulations as shown in Fig. 6. In contrast, the threshold of the proposed DMI criterion can be designed easily, since the EXIT band chart is easily obtained.

\section{Conclusion}

This paper proposed an AIDA scheme for finite-length DELDPC coded systems with MSDD. The proposed AIDA can significantly reduce the iterative decoding complexity and delay by adaptively adjusting the OWS of the MSDD SISOD and the outer iteration number between the MSDD SISOD and the LDPC decoder. In order to make AIDA effective at all SNRs, a proper SC must be used in AIDA. The existing SCs for the considered systems were analyzed, and were proved that they do not well suit for the considered systems. To overcome the disadvantages of the existing SCs, a new SC named DMI criterion was proposed based on tracking the difference of the output mutual information of the LDPC decoder between two consecutive outer iterations. Simulation results show that since the DMI criterion can effectively reflect the convergence status of the iterative decoding, compared with using the existing SCs, AIDA using the DMI criterion has better performance in terms of reducing the average number of outer iterations, performance loss and robustness.

\section{Acknowledgement}

The authors would like to thank Dr. David K. Asano of Shinshu University, for his comments and suggestions which helped improve the English of this paper. They would also like to express their sincere thanks to the Associate editor and the anonymous reviewers for their constructive and helpful comments.

\section{References}

[1] V.T. Nam, P.-Y. Kam, and Y. Xin, "LDPC codes with BDPSK and differential detection over flat Rayleigh fading channels," Proc. IEEE GLOBECOM 2007, pp.3245-3249, Nov. 2007.

[2] H. Tatsunami, K. Ishibashi, and H. Ochiai, "On the performance of LDPC codes with differential detection over Rayleigh fading channels," Proc. 63rd IEEE VTC'06, vol.5, pp.2388-2392, May 2006.

[3] M. Franceschini, G. Ferrari, R. Raheli, and A. Curtoni, "Serial concatenation of LDPC codes and differential modulations," IEEE J. Sel. Areas Commun., vol.23, no.9, pp.1758-1768, Sept. 2005.

[4] J. Li, "Differentially encoded LDPC codes-part II: General case and code optimization," EURASIP Journal on Wireless Communications and Networking, vol.2008, Article ID 367287, 2008.

[5] D. Divsalar and M.K. Simon, "Maximum-likelihood differential detection of uncoded and trellis coded amplitude phase modulation over AWGN and fading channels-Metrics and perormance," IEEE Trans. Commun., vol.42, no.1, pp.76-89, Jan. 1994.

[6] M. Peleg and S. Shamai, "Iterative decoding of coded and interleaved noncoherent multiple symbol detected DPSK," Electron. Lett., vol.33, no.12, pp.1018-1020, June 1997.

[7] V. Pauli, L. Lampe, and R. Schober, "Turbo DPSK" using soft multiple-symbol differential sphere decoding," IEEE Trans. Inf. Theory, vol.52, no.4, pp.1385-1398, April 2006.

[8] L. Wang, L.K. Kong, S.X. Ng, and L. Hanzo, "A nearcapacity differentially encoded non-coherent adaptive multiplesymbol-detection aided three-stage coded scheme," Proc. 71st IEEE VTC'10, vol.1, pp.1-5, May 2010. 
[9] L. Wang and L. Hanzo, "Multiple-symbol detection aided differential spatial division multiple access," Proc. IEEE ICC 2011, pp.1-5, June 2011.

[10] Y. Yu, S. Handa, and F. Sasamori, "Iterative multiple-symbol differential detection of differential LDPC codes," Proc. ISPACS 2010, pp.61-64, Dec. 2010.

[11] S. ten Brink, "Convergence behavior of iteratively decoded parallel concatenated codes," IEEE Trans. Commun., vol.49, no.10, pp.1727-1737, Oct. 2001.

[12] J.W. Lee and R.E. Blahut, "Generalized EXIT chart and BER analysis of finite-length turbo codes," Proc. GLOBECOM 2003, vol.4, pp.2067-2072, Dec. 2003.

[13] J. Hagenauer, E. Offer, and L. Papke, "Iterative decoding of binary block and convolutional codes," IEEE Trans. Inf. Theory, vol.42, no.2, pp.429-445, March 1996.

[14] R.Y. Shao, S. Lin, and M.P.C. Fossorier, "Two simple stopping criteria for turbo decoding," IEEE Trans. Commun., vol.47, no.8, pp.1117-1120, Aug. 1999.

[15] Y. Wu, D. Woerner, and J. Ebel, "A simple stopping criteria for turbo decoding," IEEE Commun. Lett., vol.4, no.8, pp.258-260, Aug. 2000

[16] F. Zhai and I. Fair, "New error detection techniques and stopping criteria for turbo decoding," Proc. 2000 Can. Conf. Electr. Comput. Eng., pp.58-62, March 2000.

[17] F.M. Li and A.Y. Wu, "On the new stopping criteria of iterative turbo decoding by using decoding threshold," IEEE Trans. Signal Process., vol.55, no.11, pp.5506-5516, Nov. 2007.

[18] H. Chen, R.G. Maunder, and L. Hanzo, "Low-complexity multiplecomponent turbo-decoding-aided hybrid ARQ," IEEE Trans. Veh. Technol., vol.60, no.4, pp.1571-1577, May 2011.

[19] F. Guo, S.X. Ng, and L. Hanzo, "LDPC assisted block coded modulation for transmission over Rayleigh fading channels," Proc. 57th IEEE VTC'03, vol.3, pp.1867-1871, April 2003.

[20] J. Hagenauer, "The EXIT Chart - Introduction to extrinsic information transfer in iterative processing," Proc. 12th European Signal Processing Conference, pp.1541-1548, Sept. 2004.

[21] M. Fossorier, M. Mihaljevic, and H. Imai, "Reduced complexity iterative decoding of low density parity check codes based on belief propagation," IEEE Trans. Commun., vol.47, no.5, pp.673-680, May 1999.

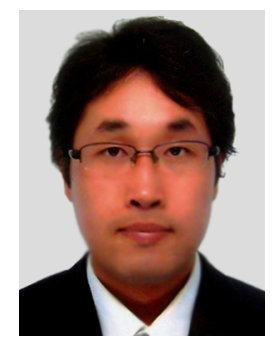

Yang Yu received the B.E. degree from Xuzhou Normal University, China in 2003, and the M.E. degree from Lanzhou University, China in 2006. He is currently pursuing the Dr.Eng. degree in the Department of Electrical and Electronic Engineering, Shinshu University. His current research interests include coding and modulation for mobile communication systems.

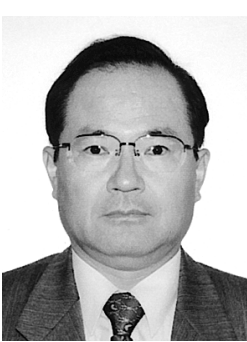

Shiro Handa received B.E. and M.E. degrees from Shinshu University in 1978 and 1980 respectively, and the Dr.Eng. degree from Kobe University in 1988. From 1982 to 1988, he was a Research Associate at Kobe University. From 1988 to 1994, he was with Nagano National College of Technology. He has been with the Department of Electrical and Electronic Engineering, Shinshu University, since 1994 as an Associate Professor and since 2005 as a professor. In 1996, he was at the University of California, Davis, as a visiting researcher. His research interests include satellite and mobile communication systems, modulation and coding, and data compression. He is a member of IEEE and SITA.

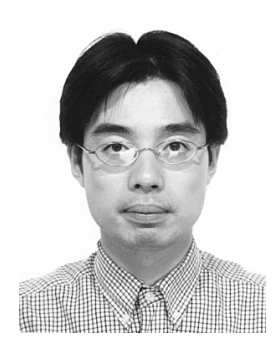

Fumihito Sasamori received the B.E., M.E. and Dr.Eng. degrees from Waseda University, Tokyo in 1994, 1996 and 2000, respectively. Since 2000, he has been with the Department of Electrical and Electronic Engineering, Shinshu University, first as a Research Associate and since 2006 as an Associate Professor. His current research interests include digital mobile communication systems. He received the IEICE Young Engineer Award in 2000. He is member of IEEE.

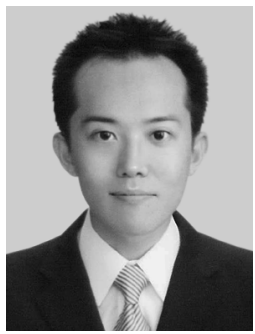

Osamu Takyu received the B.E. in Electrical Engineering from Tokyo University of Science, Chiba, Japan, in 2002, and the M.E. and Ph.D. degrees in Open and Environmental systems from Keio University, Yokohama, Japan in 2003 and 2006, respectively. From 2003 to 2007, he was a Research Associate in the Department of Information and Computer Science, Keio University. From 2004 to 2005, he was visiting scholar in the School of Electrical and Information Engineering, University of Sydney. From 2007 to 2010, he was an Assistant Professor in the Department of Electrical Engineering, Tokyo University of Science. Since 2011, he is a Research Associate in the Department of Electrical and Electronic Engineering, Shinshu University. His current research interests are in wireless communication systems and distributed wireless communication technology. He is a member of IEEE. 